Published in final edited form as:

Pain Med. 2013 December ; 14(12): . doi:10.1111/pme.12241.

\title{
Vasovagal Rates in Flouroscopically Guided Interventional Procedures: A Study of Over 8,000 Injections
}

\author{
David J. Kennedy, MD*, Byron Schneider, MD*, Ellen Casey, MD ${ }^{\dagger}$, Joshua Rittenberg, MD \\ Bryan Conrad, PhD§, Matthew Smuck, MD*, and Christopher T. Plastaras, MDף \\ *Department of Orthopaedics, Stanford University, Redwood City, California \\ ${ }^{\dagger}$ Rehabiliation Institute of Chicago, Northwestern University, Chicago, Illinois \\ ¥Kaiser Permanente, Oakland, California \\ $\S$ Nike Sport Research Lab, Beaverton, Oregon \\ IDepartment of Physical Medicine and Rehabiliation, University of Pennsylvania, Philadelphia, \\ Pennsylvania, USA
}

\begin{abstract}
Objective-To determine the rate of vasovagal (vv) complications in fluoroscopically guided interventional procedures.
\end{abstract}

Design-Retrospective case series analysis of prospectively collected data from March 8, 2004 to January 30, 2009.

Setting-A single academic medical center.

Subjects-Four thousand one hundred eighty-three subjects undergoing 8,010 consecutive injections.

Outcome Measures-Pearson's chi-square test was used to determine the relationship between categorical variables.

Results-A total of 8,010 injections, including epidural steroid injections, radiofrequency nerve ablations, medial branch blocks, hip injections, knee injections, and glenohumeral injections were performed. Overall vv reaction rate was $2.6 \%$, with $0.8 \%$ of procedures resulting in early terminated due to vv reaction. Peripheral joint injections had a vv rate of $0.2 \%$, all occurring in hip injections. Transforaminal epidural steroid injections had a vv rate of 3.5\%. Diagnostic blocks of the medial branches had the highest rate of vv (5.1\%). Other predictors of vv reactions were identified including preprocedure pain score visual analog scale $<5(P=0.004)$, male gender $(P<$ $0.001)$, and age less than 65 years old $(P<0.001)$.

Conclusions - vv reactions have an overall low occurrence rate $(2.6 \%)$ in interventional procedures ranging from $0 \%$ in peripheral knee and shoulder injections to $5.1 \%$ in medial branch blocks. Conservative treatment of vv reaction and willingness to terminate procedures resulted in no serious adverse events related to vv reaction in 8,010 procedures.

Reprint requests to: David J. Kennedy, MD, 450 Broadway Street, Pavilion C, MC 6342 Redwood City, CA 94063, USA. Tel: (650) 725-5905; Fax: (650) 723-9805; djkenned@ stanford.edu..

Disclosure: There is no conflict of interest to disclose by any of the contributing authors. 


\section{Keywords}

Interventional; Fluoroscopy; Zygoapophyseal Joint; Sacroiliac Joint; Physician Performance; Medial Branch; Facet Joint

\section{Introduction}

Spine and appendicular pain complaints are on the rise. Predictably, the rates of imageguided interventions for these conditions have also increased, but at a more rapid pace. For instance, between 1994 and 2001, the utilization of lumbar epidural corticosteroid injections increased by $241 \%$ in the United States Medicare population [1].

Overall, these interventions have very low complication rates ranging from $1 \%$ to $12 \%$, depending upon how complications were identified and defined. vv reactions during spine injections are frequently cited as a common immediate adverse event with a range of reported rates between $0 \%$ and $8.7 \%$ [2-13]. Typical symptoms for vasovagal (vv) reactions include: lightheadedness, dizziness, palpitations, weakness, dimming or blurred vision, nausea and epigastric distress, feeling warm or cold, facial pallor, sweating, dilated pupils, and syncope [14]. In addition to these unpleasant but relatively benign symptoms, other negative consequences of $\mathrm{vv}$ reactions include aborted procedures and even asystole in severe cases $[7,9,15]$. To the best of the authors' knowledge, the comparative rates of $\mathrm{vv}$ reactions during various image-guided spine and peripheral joint interventions have not been reported, and risk factors for $\mathrm{vv}$ reactions are ill-defined in this population. The goal of this study is to determine the comparative frequency of $\mathrm{vv}$ reactions in multiple types of interventional procedures and to investigate factors that predispose this population to $\mathrm{vv}$ reactions.

\section{Methods}

This study is a retrospective analysis of an existing prospectively collected dataset including 6,116 consecutive patients treated at a single academic medical center between 2004 and 2008. This study was institutional review board approved and Health Insurance Portability and Accountability Act compliant. All interventions were performed using fluoroscopicguidance in either an office-based fluoroscopy suite or ambulatory surgery center by one of four experienced physicians with and/or without trainee involvement. The treating physicians were all board-certified in Physical Medicine and Rehabilitation and had additional subspecialty certification in either Sports Medicine or Pain Medicine. In the selected cases that involved moderate sedation, patients were asked to observe nil per Os (NPO) precaution starting at midnight, the night prior to the injection. Only patients that received moderate sedation had an intravenous (IV) placed prior to the procedure, and none received preprocedural IV fluids. During the procedure, all patients were actively monitored via continuous pulse oximetry and intermittent automatic blood pressure monitoring by a registered nurse positioned at the head of the bed whose sole responsibility was to monitor the patient. Patients were noted to have a vv reaction by the attending physician if they had a decrement in heart rate and blood pressure as well as symptoms consistent with vv reaction including: lightheadedness, dizziness, palpitations, weakness, dimming or blurred vision, nausea and epigastric distress, feeling warm or cold, facial pallor, excessive sweating, and syncope. If the patient were noted to have these symptoms in the recovery area, the physician was notified, and a vv reaction was documented as well.

Per standard protocol, immediately after the intervention, the treating physician entered all data into a single database using preset drop-down menu choices to facilitate standardized 
reporting. Baseline demographic, and multiple clinical and procedural characteristics were noted, including age, gender, preprocedure and postprocedure pain scores, type of procedure and target level(s), needle gauge, needle length, fluoroscopy time, termination before completion, and complications. Each of the following complications was included in the drop-down menu choices: vv reaction, intravascular injection, hypertension, intolerable pain, tachycardia, dural puncture, and allergic reaction. vv reactions were noted only if the reaction occurred following the start of the procedure and before the patient was transferred from the treatment room to recovery. Procedure termination was at the sole discretion of the attending physician.

Statistical analyses were done per injection, rather than per patient. To determine the relationship between categorical variables, Pearson's chi-square test was used. The assumption that the sampling distribution of each variable approximated a chi-square distribution was checked by ensuring that the expected frequencies in each cell were at least five. In situations where sample sizes are less than five, the Fisher's exact test was used. All statistical analysis was performed using SPSS version 20 (IBM Corp., Armonk, NY, USA). Significance values were set $a$ priori at a level of $P<0.05$.

\section{Results}

The overall rate of a vv reaction was $2.6 \%$ of the injections, with early termination due to vv recorded in $0.8 \%$ of those injections. In total, 8,010 injections were recorded on the 4,183 consecutively treated patients, with 4,940 injections performed on females (age: $56.1 \pm 16.8$ years) and 3,067 performed on males (age: $54.4 \pm 16.5$ ). Please refer to Table 1 for details of the procedure.

While rates of vv reactions were not significantly different between the different procedure types, some interesting trends are observed. Collectively, transforaminal epidural injections accounted for $54 \%$ of the procedures performed and $74.8 \%$ of the vv reactions, with a vv rate of $3.5 \%$ (Table 2). Peripheral joint injections including hip, knee, and glenohumeral accounted for 1,428 procedures and collectively had a vv rate of $0.2 \%(\mathrm{~N}=3)$. Of the intraarticular injections, only the hip joint had any documented vv reactions at a rate of $0.5 \%$. There were no documented vv reactions for intra-articular knee or shoulder injections despite large numbers of these in the dataset. However, subacromial bursa injections had a rate of $1.6 \%$. Medial branch and dorsal ramus anesthetic blocks demonstrated a vv reaction rate of $5.1 \%$, while radiofrequency neurotomy of these same nerves had a rate of only $1.35 \%$.

The following variables were individually analyzed for their association to vv episodes: age, gender, needle length, needle gauge, fluoroscopy time, procedure, and preprocedure and postprocedure pain scores. Of these, gender, age, preprocedure pain score, and needle gauge were each found to have significant associations. Specifically, males were 2.0 times as likely to experience a vv episode compared with females (3.8\% vs $1.9 \%$, relative risk $=2.4, P<$ $0.001, \chi^{2}=29.287, \mathrm{df}=1$, Table 3 ). With a rate of $3.3 \%$ in patients under 65 compared with $1.1 \%$ in those over 65 , patients under the age of 65 had a 2.4 times higher odds $(P<0.001$, $\chi^{2}=30.622, \mathrm{df}=1$, Table 4 ) of experiencing a vv episode. Finally, patients with a preprocedure pain less than 5 on a $0-10$ numeric pain rating scale had a vv reaction rate of $3.2 \%$ compared with $2.2 \%$ for those with preprocedure pain greater than $5(P=0.004)$. Analyzing for all injections, needle gauge was significantly associated ( $3.0 \%$ for 22 gauge, $1.6 \%$ for 25 gauge, $0.4 \%$ for 20 gauge; $P<0.001$ ). No vv events occurred with use of 18 gauge needle. Overall, there was also a significant effect of needle length, but this was no longer significant after controlling for injection type. That is, for each type of injection, there was no association between needle length and likelihood of vv. 
Given the large predominance of transforaminal epidural steroid injections (TFESIs) within our cohort, we also evaluated cervical vs lumbar/sacral level for association with vv. Only $1 / 245(0.41 \%)$ cervical level TFESIs were complicated by a vv event compared with $154 / 4,201(3.67 \%)$ vv events during lumbar or sacral TFESIs $\left(\chi^{2}=7.30, P<0.007\right)$.

\section{Discussion}

This study of a large consecutive cohort of over 4,000 patients and 8,000 injections provides new insights into the risk factors associated with vv events and their comparative rates between 22 common interventions. This study shows that the rate of a vv reaction increases with medial branch blocks, male sex, younger age, and lower preprocedural pain scores. It has previously reported that patients who are mildly hypovolemic are more susceptible to cardiovascular depression with thoracic peridural anesthetic blocks [16]. However, none of the patients that were NPO prior to their procedure experienced a vv event or any other cardiovascular complication. Approximately $30 \%$ of those with a documented vv reaction required early termination of the procedure. Treatment for the $\mathrm{vv}$ reactions consisted of placing the patient in Trendelenberg position with a cool and damp cloth applied to the neck. No patients required oral medications, IV medication, IV fluid bolus, or transportation outside the surgery center or clinic. Fortunately, no serious or catastrophic complications were recorded during these procedures or in any of the patients in this large cohort. In this cohort of over 8,000 procedures, none of the patients required transportation to the hospital for a procedural complication. In fact, only one patient in this group required transfer to an acute care facility. That patient had a preprocedural hypertensive emergency that was noted on nursing intake, and he never actually underwent the procedure.

To the best of the authors' knowledge, this is the first study to directly compare vv rates between various percutaneous axial and appendicular procedures. The observed $3.5 \%$ rate of vv reactions for transforaminal epidural injections is consistent with published reports [2$6,8,9]$. This indicates that the relative rates documented between injection types in this study, while specific to a single center, likely reflect those in general practice. This study shows great variability in rates of vv reactions based on procedure type from $0 \%$ for intraarticular shoulder and knee joint injections to $5.1 \%$ for medial branch blocks. In fact, appendicular injections were nearly void of vv reactions, the only exception being intraarticular hip joint injections (0.5\%) and subacromial bursa injections (1.65).

Interestingly, medial branch blocks resulted in a vv rate of $5.1 \%$, while radiofrequency neurotomy had a lower rate of $1.35 \%$. This discrepancy between procedures may help account for the previously reported variability in interventional procedures between $0 \%$ and $8.7 \%$ [2-12]. A higher rate with medial branch blocks relative to other axial and appendicular interventions is not surprising because medial branch blocks require needle insertion and advancement to at least two target sites while the others commonly involve a single target. What is surprising is that this same trend was not observed for radiofrequency neurotomies, which also has multiple targets. There was not a significant difference in patient characteristics between the two groups. The rates could be due to the longer time required to complete the procedure. The total procedural time was not recorded. Even if it were noted, this data may be flawed given that approximately $30 \%$ of patients with a vv reaction had the procedure terminated early, thus artificially lowering procedural time. Also of interest was the fact that cervical transforaminal epidural injections had a statistically significant lower rate of vv reactions than lumbar transforaminal epidural injections $(0.41 \%$ vs $3.67 \%, P<0.007)$. The reason for this is unclear and has not been previously reported in the literature. It may be due to anatomic features such as cervical injections being relatively more superficial than their lumbar counterparts. The standard needle used for a cervical transforaminal epidural was a 25 -gauge, 2 -inch needle. This needle was never utilized in the 
lumbar spine, with those procedures requiring a minimum of a 3.5 -inch needle, thus negating the ability of a direct comparison. Also, trainee involvement may have been a factor as fellows typically only started doing cervical procedures once they had demonstrated competence in lumbar procedures.

There were several baseline demographics that also correlated with increased likelihood of developing a vv reaction including: male sex, younger age, and lower preprocedural pain scores. The underlying mechanism for these findings are unclear. While one author of this study has suggested to the others that a gender difference in fortitude underlies this finding, the majority dispute this explanation. Alternative explanations include that: vasodilation in the setting of a greater muscle mass may produce a more precipitous decrease in blood pressure, lower baseline pain scores allows for a greater percentage rise in pain with greater consequences, and adults over 65 may have experienced more pain and have more experience with previous medical interventions.

There are several limitations to this study. First, due to practice patterns, there were limited numbers of some procedures, such as discography, and several others that were not performed in this cohort including: spinal cord stimulator trials, verterbroplasty, kyphoplasty, sympathetic blocks, and sacral lateral branch blocks. Because the vast majority, $97.3 \%(\mathrm{~N}=7,796)$, of the injections were performed without moderate sedation, this variable was not included in the analysis. Also, there were a number of physicians performing the procedures, sometimes including trainee involvement. The effects of trainee involvement on vv events and other complications during injections have not been previously reported in the literature. As such, the effects of trainee involvement effects on injections is a potential confounding variable as well as a topic that should be explored in future research. Variations in technique may have resulted in significant differences in rates between physicians that were not assessed in this study. Another limitation was in the definition of a vv reaction. In this study, a vv reaction was recorded when the patient had a decrement in blood pressure and heart rate in addition to symptoms consistent with a vv reaction. This was done at the discretion of the attending physician and did not require a specific numerical change in blood pressure or heart rate. This could have resulted in either an overestimation or underestimation of the rate of vv reaction. Lastly, this data was reviewed retrospectively, although it was entered in a strict prospective manner utilizing an electronic medical record with drop-down menu choices to facilitate ease of data entry at the time of the procedure.

\section{Conclusions}

Vv reactions have an overall low occurrence rate in fluoroscopic-guided percutaneous interventions with variability between procedures, from none in knees and shoulders to $3.5 \%$ in transforaminal epidural injections, and 5.1\% in medial branch blocks. vv reactions are more prevalent in males, in those under 65 years of age, and in those with preprocedure pain scores $<5 / 10$. vv reactions occur more frequently in lumbrosacral TFESIs compared with cervical TFESIs. Early termination of the procedure occurs in $30 \%$ of cases involving a vv reaction. With rapid identification and simple conservative treatments along with a willingness to terminate the procedure, there were no serious complications in this cohort and no need for medication administration. It is recommended that patients are actively monitored during these procedures for prompt recognition of any adverse reaction and if necessary, the procedure be terminated to avoid further complications. 


\section{References}

1. Friedly J, Chan L, Deyo R. Increases in lumbosacral injections in the medicare population. Spine. 2007; 32(16):1754-60. [PubMed: 17632396]

2. Cicala RS, Westbrook L, Angel JJ. Side effects and complications of cervical epidural steroid injections. J Pain Symptom Manage. 1989; 4(2):64-6. [PubMed: 2732522]

3. Karaman H, Kavak GO, Tufek A, Yldrm ZB. The complications of transforaminal lumbar epidural steroid injections. Spine. 2011; 36(13):E819-24. [PubMed: 21217425]

4. Manchikanki L, Malla Y. A prospective evaluation of complications of 10,000 fluoroscopically directed epidural injections. Pain Physician. 2012; 15:131-40. [PubMed: 22430650]

5. Botwin KP, Gruber RD, Bouchlas CG, et al. Complications of fluoroscopically guided transforaminal lumbar epidural injections. Arch Phys Med Rehabil. 2000; 81(8):1045-50. [PubMed: 10943753]

6. Huston CW, Slipman CW, Garvin C. Complications and side effects of cervical and lumbosacral selective nerve root injections. Arch Phys Med Rehabil. 2005; 86(2):277-83. [PubMed: 15706554]

7. Florida N. Quality assurance for interventional pain management procedures in private practice. Pain Physician. 2008; 11(1):43-55. [PubMed: 18196169]

8. Waldman S. Complications of cervical epidural nerve blocks with steroids: A prospective study of 790 consecutive blocks. Reg Anesth. 1989; 14(3):149-51. [PubMed: 2486595]

9. Botwin K, Gruber R, Bouchlas C, et al. Complications of fluoroscopically guided caudal epidural injections. Am J Phys Med Rehabil. 2001; 80:416-24. [PubMed: 11399002]

10. Plastaras CT, Joshi AB, Garvan C, et al. Adverse events associated with fluoroscopically guided sacroiliac joint injections. PM \& R. 2012; 4(7):473-8. [PubMed: 22543036]

11. Ahmed SU, Tonidandel W, Trella J, Martin NM, Chang Y. Peri-procedural protocols for interventional pain management techniques: A survey of US pain centers. Pain Physician [Internet]. 2005; 8(2) Available at: http://www.painphysicianjournal.com/2005/april/ 2005;8;181-185.pdf.

12. Pobiel R, Schellhas K, Eklund J, et al. Selective cervical nerve root blockade: Prospective study of immediate and longer term complications. AJNR Am. J. Neuroradiol. 2009; 30:507-11. [PubMed: 19193762]

13. Manchikanti L, Malla Y, Wargo BW, et al. Complications of fluoroscopically directed facet joint nerve blocks: A prospective evaluation of 7,500 episodes with 43,000 nerve blocks. Pain Physician. 2012; 15(2):E143-50. [PubMed: 22430660]

14. Wieling W. Reflex syncope in children and adolescents. Heart. 2004; 90(9):1094-100. [PubMed: 15310717]

15. Stauber B, Ma L, Nazari R. Cardiopulmonary arrest following cervical epidural injection. Pain Physician. 2012; 15:147-52. [PubMed: 22430652]

16. Bonica JJ, Kennedy WF, Akamatsu TJ, Gerbershagen HU. Circulatory effects of peridural block: 3. Effects of acute blood loss. Anesthesiology. 1972; 36(3):219-27. [PubMed: 5011415] 


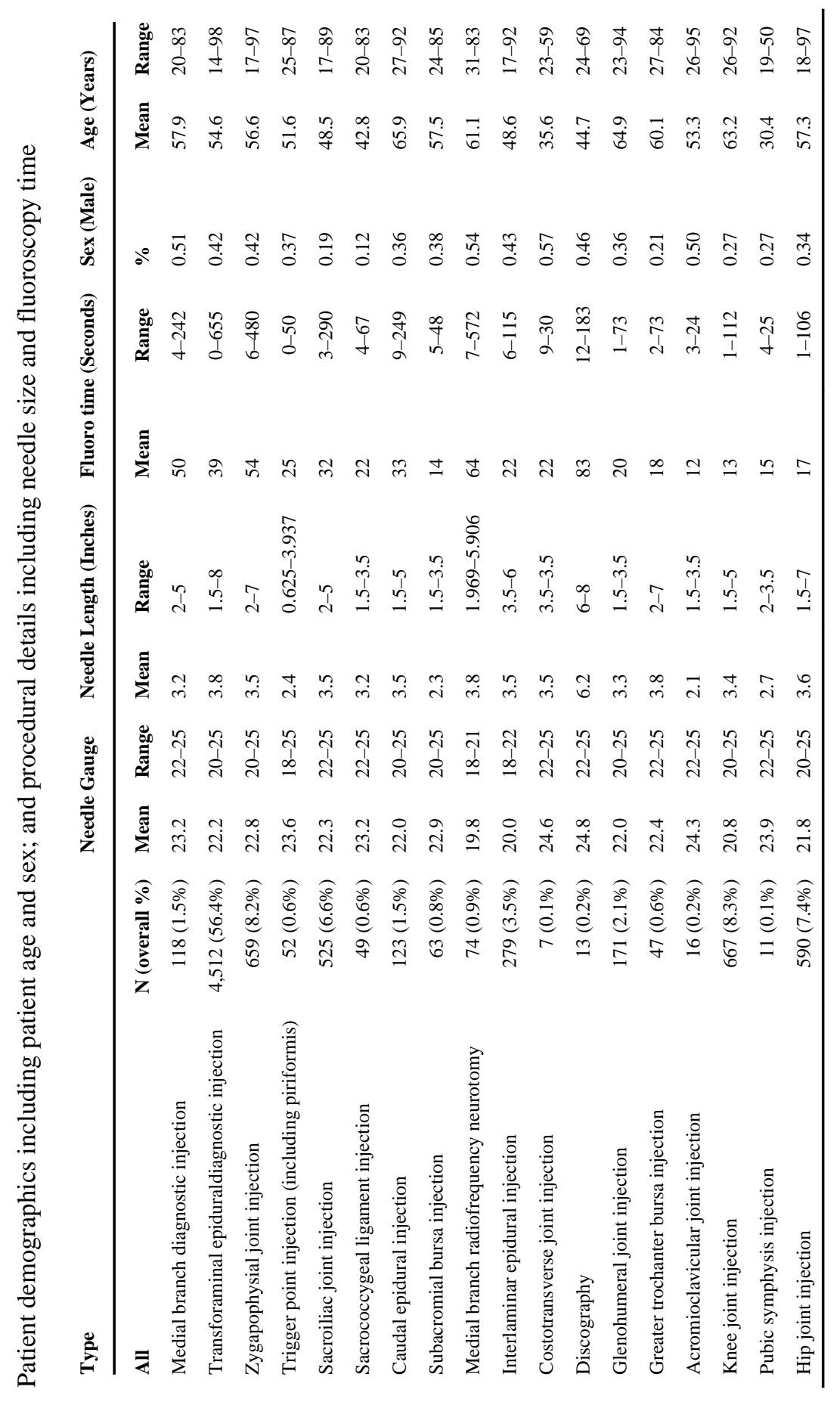

Pain Med. Author manuscript; available in PMC 2014 March 01. 
Table 2

Type of injection and percentage resulting in vasovagal reaction

\begin{tabular}{ll} 
Injection Type & \% Resulting in Vagal \\
\hline Medial branch diagnostic injection & 5.10 \\
Transforaminal epidural injection & 3.50 \\
Diagnostic selective spinal nerve injection & 3.30 \\
Zygapophysial joint injection & 3.30 \\
Trigger point injection & 2.60 \\
Sacroiliac joint injection & 2.50 \\
Sacrococcygeal ligament injection & 2.00 \\
Caudal epidural injection & 1.60 \\
Subacromial bursa injection & 1.60 \\
Radiofrequency neurotomy & 1.35 \\
Interlaminar epidural injection & 0.70 \\
Femoral-acetabular joint injection & 0.50 \\
Acromioclavicular joint injection & 0.00 \\
Costotransverse joint injection & 0.00 \\
Discography & 0.00 \\
Glenohumeral joint injection & 0.00 \\
Greater trochanter bursa injection & 0.00 \\
Joint injection & 0.00 \\
Knee joint injection & 0.00 \\
Piriformis muscle injection & 0.00 \\
Pubic symphysis injection & 0.00 \\
Soft tissue injection & 0.00 \\
\hline
\end{tabular}




\section{Table 3}

Contingency table for vasovagal episodes and gender

\begin{tabular}{ccccc} 
& \multicolumn{2}{c}{ Gender } & \\
\cline { 3 - 4 } & & Female & Male & Total \\
\hline Vagal & No & $4,848(60.5 \%)$ & $2,949(36.8 \%)$ & $7,800(97.4 \%)$ \\
& Yes & $92(1.1 \%)$ & $118(1.5 \%)$ & $210(2.6 \%)$ \\
Total & & $4,940(61.7 \%)$ & $3,067(38.3 \%)$ & 8,010 \\
\hline
\end{tabular}

Cells represent counts of observations in each category. In three cases, gender was not specified. 
Table 4

Contingency table for vasovagal episodes and age

\begin{tabular}{ccccc} 
& \multicolumn{2}{c}{ Age } & \\
\cline { 2 - 4 } & & Under 65 & Over 65 & Total \\
\hline \multirow{2}{*}{ Vagal } & No & $5,412(67.6 \%)$ & $2,388(29.8 \%)$ & $7,800(97.4 \%)$ \\
& Yes & $183(3.3 \%)$ & $27(0.3 \%)$ & $210(2.6 \%)$ \\
\multirow{2}{*}{ Total } & & $5,595(69.9 \%)$ & $2,415(30.1 \%)$ & 8,010 \\
\hline
\end{tabular}

Cells represent counts of observations in each category. 